\title{
p53/Surviving Ratio as a Parameter for Chemotherapy Induction Response in Children with Acute Myeloid Leukemia
}

Rinaldi Lenggana ${ }^{1,4^{*}}$, Susanto Nugroho ${ }^{2,4}$, Sri Winarsih ${ }^{3}$

\author{
${ }^{1}$ Biomedical Science Master Study Program, Faculty of Medicine, Brawijaya University, Malang, Indonesia \\ ${ }^{2}$ Department of Paediatrics, Faculty of Medicine Brawijaya University, Malang, Indonesia \\ ${ }^{3}$ Pharmacology Department, Faculty of Medicine, Brawijaya University, Malang, Indonesia \\ ${ }^{4}$ Saiful Anwar Public Hospital, Malang, Indonesia
}

\begin{abstract}
Acute myeloid leukemia (AML) is a malignancy that is often found in children. Many studies into the failure of apoptosis function, or programmed cell death, is one of the most important regulatory mechanisms of cellular hemostasis which is closely linked to the development of cancer, are important. Also, regulation of the apoptotic (p53) and anti-apoptotic (surviving) proteins influence treatment outcome. One role of p53 is to monitor cellular stress necessary to induce apoptosis. Surviving (BIRC5) is a group of proteins in the apoptosis inhibitor which works by inhibiting caspase-3. The role of surviving is considered very important in oncogenesis proliferation and cell growth regulation. Chemotherapy in childhood AML can inhibit cell growth and induce slowing as well as stopping the cell cycle. Thus, the aim of this study was to compare p53 and surviving before and after receiving induction chemotherapy in children with AML and also to determine the p53/surviving ratio. Peripheral blood mononuclear cells were collected from AML children before treatment and three months after starting their induction therapy. p53 and surviving were measured by flowcytometry using monoclonal antibodies. Data were analyzed by t-test for comparison between groups and Spearman's test to find out the correlation between variables with a significant value of $\mathrm{p}<0.05$. A total of 8 children were evaluated. The intensity of $\mathrm{p} 53$ expression was not significantly increased after induction phase chemotherapy $(\mathrm{p}=0.224)$, but surviving expression and the ratio of $\mathrm{p} 53 /$ surviving were significantly increased in the treatment group compared with the levels prior to chemotherapy $(p=0.002, p=0.034)$, and there was a strong negative correlation between $p 53$ and surviving after chemotherapy $(\mathrm{r}=-0.63, \mathrm{p}=0.049)$.
\end{abstract}

Keywords: p53, surviving, acute myeloid leukemia

\section{INTRODUCTION}

Leukemia is a malignant disease in children with the highest incidence between the ages of 2-5 years [1] and is the most malignancy in children with an incidence of 4-4.5 cases/year/100,000 [2]. In Europe, in the period between 1988 -1997, acute leukemia incidence was 22.6 per one million of which $15 \%$ was AML. In Germany, the proportion of AML was $13 \%$ while in the US, it was $16 \%$. In Yogyakarta, the incidence of ALL was 20.8/1,000,000 while for AML it was $8 / 1,000,000$. AML mainly occurs from 2-5 years [3].

\section{${ }^{*}$ Corresponding author:}

Rinaldi Lenggana

Biomedical Science Master Study Program, Faculty of Medicine, Brawijaya University

Jalan Veteran, Malang, Indonesia 65145

E-mail: aldilenggana@gmail.com
But AML Treatment has made much progress in developed countries with success reaching $65 \%$, whereas, in Indonesia, it is expected to remain below 10\% [3].

The mechanism of apoptosis, which is a process of cell death, attempts to remove unwanted and excess cells. Thus, apoptosis is important for protection against pathogens and tissue homeostasis [4].

One role of p53 is to monitor for cellular stress and for its resulting damage which can be severe and irreparable. Tumor suppressors act to maintain tissue homeostasis and to control the number and behavior

How to cite:

Lenggana R, Nugroho S, Winarsih S (2016) p53/Surviving Ratio as a Parameter for Chemotherapy Induction Response in Children with Acute Myeloid Leukemia . J. Trop. Life. Science 6 (3): $142-150$. 
of cells in certain tissues and p53 is a suppressor that has been widely studied which acts in response to various forms of cellular stress and is involved in approximately $50 \%$ of human cancers [5]. The loss of p53 function causes genomic instability and increased mutagenesis, and it is thought that the mutation of p53 is one of the factors that cause cancer resistance to chemotherapy [6].

Surviving (BIRC5) is a therapeutic target because its expression occurs during the development of malignancy and its role is considered to be important both in oncogenesis and in cell proliferation and growth regulation [7]. Physiologically, surviving is detected in high levels in the fetal period, but declines as the fetus develope. When the surviving level was increase, it should be correlates with the incidence of relapse and treatment failure and the low life expectancy of patients with AML[7, 8].

The expression of surviving in solid tumors has seen many studies and in theory, there is a relationship between p53 as a pro-apoptosis and surviving as an inhibitor of apoptosis [8]. Thus, this research hoped it could be used as a reference for the ratio of p53/surviving that can be used as a prognostic marker for AML treatment in children. AML chemotherapy in children can result in DNA damage which can stimulate p53 to inhibit surviving expression [9].

Therefore, the aim of this study was to measure the ratio p53/surviving to investigate its use as a parameter in the induction phase chemotherapy response in children with acute myeloid leukemia.

\section{MATERIALS AND METHODS}

The study used a study design with a pre and postexperiment approach (cohort study). Sampling was done by consecutive sampling and this research was conducted at the Department of Pediatrics Dr. Saiful Anwar Hospital, Malang and the Biomedical Laboratory of the Faculty of Medicine, Brawijaya University. Sampling was conducted from March 2015 to August 2015, and the subjects were patients under treatment in the Department of Pediatrics Dr. Saiful Anwar Malang Hospital. The criteria for the diagnosis of AML was based on morphological analysis of bone marrow punctures. The study subjects were then divided into complete remission, partial remission and no remission groups. Informed consent was obtained from all the relevant parents.

Criteria for the inclusion of cases was: 1 . AML patients, 2.post induction phase chemotherapy 3. age below 14 years, 4.informed consent from relevant par- ents, and the exclusion criteria were: 1 . patients with drug withdrawal during the induction phase of chemotherapy and 2. autoimmune diseases, such as SLE and myasthenia gravis. This study has been approved by the Ethics Committee of Saiful Anwar Hospital.

Peripheral blood mononuclear cells (PBMC) were isolated from EDTA blood by Ficoll-Hypaque density gradient centrifugation. Intracellular staining of protein was performed according to the manufacturer's instructions. Phycoerythrin (PE) labeled anti-surviving monoclonal antibodies were provided by $\mathrm{R} \& \mathrm{D}$ System Inc. (catalog \#:IC6472P), and fluorescein isothiocyanate (FITC) labeled p53 monoclonal antibodies were obtained from Santacruz (catalog \#:sc7272FITC). Briefly, cells were incubated in the dark at $18-240 \mathrm{C}$ for 15 minutes. Cells were treated with a cell staining buffer in each sample and centrifuged at $500 \mathrm{G}$ for 5 minutes. Then, the Cells then were fixed by fixation buffer in the dark at $18-240 \mathrm{C}$ for 20 minutes and centrifuged at $500 \mathrm{G}$ for 5 minutes. Cell pellets were washed with permeabilization buffer. They were then incubated with a cocktail of FITC p53 and PE anti-surviving in the dark at room temperature for 30 minutes. All the labeled cells were resuspended in $0.5 \mathrm{~mL}$ cell staining buffer and analyzed by flowcytometry using the FACSCalibur instrument (Becton Dickinson) and the Cell Quest software package (Becton Dickinson). In this study, data analysis techniques were calculated in three stages, respectively: (1) Normality test of data samples with Shapiro-Wilk test, (2) T-test was done for comparison between groups (3) Spearman's Test to find out the correlation between variables. Statistical analysis of data was performed by SPSS software for Windows 22.0. The data are presented as mean \pm standard deviations.

\section{RESULTS AND DISCUSSION}

All research subjects had been diagnosed with AML, then the expression levels of p53 and surviving were examined. After which, the subjects were administered the appropriate chemotherapy by TRAP/ COAP/POMP protocols conducted by standard operating procedures of the Hematology-oncology Department in Saiful Anwar Hospital. The induction phase was carried out over three months, and then bone marrow puncture was re-done to see the results of chemotherapy response (complete remission, partial remission or no remission). Expressions of p53 and surviving were obtained by using flowcytometry conducted at the Biomedical Laboratory, Brawijaya University. All eight children received induction phase chemotherapy 
Table 1. Results of Research Subjects

\begin{tabular}{lc}
\multicolumn{1}{c}{ Notes } & Subject $(n=8)$ \\
\hline Sex & \\
- Male & $5 / 8$ \\
-Female & $3 / 8$ \\
Age (months) & $52.87 \pm 6.6$ \\
AML type & \\
- M 1 & $1 / 8$ \\
- M 2 & $3 / 8$ \\
- M 3 & $0 / 8$ \\
- M 4 & $4 / 8$ \\
- M 5 & $0 / 8$ \\
- M 6 & $0 / 8$ \\
- M 7 & $0 / 8$ \\
Induction phase results & \\
- Complete remission & $7 / 8$ \\
- Partial remission & $1 / 8$ \\
- No remission & $0 / 8$ \\
\hline
\end{tabular}

and their levels of p53 and surviving were noted and before and after the chemotherapy.

The results are shown in Table 1.

Study results indicated that $50 \%$ of subjects suffered from type M4 AML, 37.5\% had type M2 AML, and $12.5 \%$ suffered from type M1 AML. After being given the induction phase chemotherapy, 7 out of 8 (88\%) experienced complete remission and 1 out of 8 (12\%) experienced partial remission.

Based on flowcytometry figures, the expressions of p53 and surviving were analyzed and the ratio of p53/surviving calculated (Table 2). The p53 expression was processed by descriptive analysis and paired T-test. In all 8 of the research subjects, comparative values obtained were p53 $>\mathrm{R} 2=3.24 \%$ so that it can be interpreted that chemotherapy affects approximately $3.24 \%$ of p53 expression. The significance level of the effect of chemotherapy on $p 53$ was $p=0.224(p>0.05)$, the expression of $\mathrm{p} 53$ after the induction phase chemotherapy was not significantly increased.

Comparison of the results of paired T-test, found that surviving $\rightarrow \mathrm{R} 2=62.25 \%$, chemotherapy affected $62.25 \%$. The significance level of the effect of chemotherapy on surviving was $\mathrm{p}=0.02(\mathrm{p}<0.05)$ and the expression of surviving after the induction phase chemotherapy was significantly decreased.

A paired T-test obtained a comparative ratio of p53/surviving before and after induction phase of chemotherapy with a value of $p=0.034(p<0.05)$. i.e. the $\mathrm{p} 53 /$ surviving ratio after induction phase chemotherapy was significantly increased.

Spearman correlation test obtained a correlation co- efficient of -0.63 , which means that this study's results found a strong correlation with the direction of a negative correlation: the higher the variable of the p53 expression, the lower the surviving expression. The result obtained from the Spearman correlation test indicated a significance value of $\mathrm{p}=0.049(\mathrm{p}<0.05)$, i.e. a negative one.

Based on Table 3, the study subjects showed increased levels of p53 after their chemotherapy. However, the p53 expression had increased but not significantly because one subject had decreased levels of p53 after the induction phase chemotherapy. In networks where stressors produce severe damage which can not be repaired, p53 can initiate apoptosis so as to eliminate the damaged cells [10]. Also, tumor suppressors act to maintain tissue homeostasis to control the number and behavior of cells in the body. p53 is a muchstudied tumor suppressor which acts in response to various forms of cellular stress to mediate antiproliferative processes. Therefore, p53 can be activated by DNA damage, hypoxia or aberrant expression of oncogenes that drive changes in the cell cycle, DNA repair, cellular senescence, and apoptosis. By the p53's potential role in modulating cancer chemotherapy, the loss of p53 function is associated with chemotherapy resistance in certain tumor types [11]. Thus, for a complete understanding of the apoptotic program it is hoped that research into p53 will lead to better diagnoses and thus to better prognoses as well as to improvements in therapy $[10,12]$.

In this study there was one sample which was not consistent with the hypothesis, decreased levels of p53 after the induction phase chemotherapy. It was probably caused by the complexity of the apoptotic pathways themselves [10]. The inhibition of proteosome can cause decreased p53 levels together with the overexpression of surviving that is mediated by MDM-2 caspase. This explains that homologous of p53 such as DNp63, Tap73, dan DNp73 in mRNA are related to surviving levels [13]. There are two main pathways of apoptosis, first, the extrinsic pathway (death receptor pathways) and second, the intrinsic pathway (mitochondrial pathways). These two pathways end with the activation of caspase (the enzyme that effects cell death) $[12,14]$. Lose of p53 function related to chemotherapy resistence in several tumor types.

The stimulation of receptor death cells will result in aggregation and the recruitment of adaptor Fas-associated protein with death domain (FADD) and caspase8. After which, the caspase- 8 will be activated and start the process of apoptosis by the caspase effector [15]. 
Table 2. p53 and surviving expression results before and after the induction phase chemotherapy.

\begin{tabular}{ccccccc}
\hline Subject & $\begin{array}{c}\text { p53 before chemotherapy } \\
\text { (\%) }\end{array}$ & $\begin{array}{c}\text { p53 after } \\
\text { chemotherapy } \\
(\%)\end{array}$ & $\begin{array}{c}\text { Surviving before } \\
\text { chemotherapy } \\
(\%)\end{array}$ & $\begin{array}{c}\text { Surviving after } \\
\text { chemotherapy } \\
(\%)\end{array}$ & $\begin{array}{c}\text { p53/Surviving } \\
\text { Before } \\
\text { chemotherapy }\end{array}$ & $\begin{array}{c}\text { p53/Surviving } \\
\text { after } \\
\text { chemotherapy }\end{array}$ \\
\hline 1 & 56.38 & 99.28 & 96.16 & 55.26 & 0.58 & 1.79 \\
2 & 62.21 & 86.74 & 59.41 & 38.20 & 1.04 & 2.27 \\
3 & 45.14 & 79.85 & 44.23 & 22.01 & 1.02 & 3.62 \\
4 & 56.18 & 77.61 & 58.20 & 48.49 & 0.96 & 1.6 \\
5 & 60.53 & 63.75 & 80.45 & 68.57 & 0.75 & 0.93 \\
6 & 72.71 & 79.54 & 99.86 & 63.54 & 0.73 & 1.25 \\
7 & 99.92 & 55.92 & 89.04 & 58.05 & 1.12 & 0.96 \\
8 & 24.58 & 35.40 & 60.20 & 53.54 & 0.4 & 0.66 \\
\hline
\end{tabular}

The Mitochondria is involved in intrinsic pathways through stress stimulation including UV radiation, Cradiation, heat, DNA damage, oncoprotein activators, chemotherapy and p53 $[13,16]$. The permeability of mitochondrial membranes depends on the stability of the activity between pro-apoptoses such as sitokrom c, Smac/Diablo, Omi/HtrA2 (caspase-dependent), AIF and Endo G (non-caspase-dependent) and anti-apoptosis protein such as $\mathrm{Bcl} 2$ ( $\mathrm{Bax}, \mathrm{Bak}, \mathrm{Bcl} 2$ dan $\mathrm{Bcl}-\mathrm{XL}$, Mcl-1) [17]. p53 plays a role in the regulation of proapoptosis in the nucleus.

The initiator caspase can directly activate pro-caspase- 3 or other executor caspase that result in apoptosis. Also, the initiator caspase can activate Bid, a protein that can react with the mitochondrial membrane to stimulate cytochrome-c which activates caspase-9 and caspase-3 [18].

Chemotherapy initiates apoptosis pathways both extrinsically and intrinsically, and chemotherapeutic agents which cause DNA damage activate p53 from the inhibition of MDM-2 [19]. In the extrinsic pathway, p53 enables to capture of the death receptor signal, further inducing procaspase- 8 to caspase- 8 and procaspase- 3 into caspase- 3 to trigger apoptosis. In the intrinsic pathway, the mitochondrial membrane permeability is regulated by a balance between protein proapoptosis (Bid and Bax) and antiapoptosis proteins (Bcl-2) [16] [20]. The activation of Bax, caspase- $2 \mathrm{tBid}$ and leads to impaired mitochondrial membrane permeability and the release of cytochrome-c which then activates the apoptotic cascade. Cytochrome-c binds to procaspase-9 and Apaf- 1 to activate caspase-9. Also, inhibition of the Bcl-2 action, also causes p53 to inhibit surviving (inhibitor of apoptosis proteins) so that the process of apoptosis via caspase- 9 and caspase- 3 continues. Caspase-12, which is activated by the endoplasmic reticu- lum stress, causes direct activation of caspase-9 and then activates caspase- 3 as an effector caspase [21]. A study by Hui et al., 2010, found that the presence of a positive correlation between the levels of surviving and p53 in cases of liver malignancy [22], the results of which were also similar to those of a study by Baytekin et al., 2011, in kidney malignancies (renal cell ca) which discovered high levels of surviving in cases resistant to chemotherapy [23].

Based on Table 4, all study subjects showed decreased expressions of surviving after the induction phase chemotherapy, this is significant and conforms to the hypothesis of this study. Significant levels of surviving have been found in patients with such cancers like lung, genito-urinary, stomach, colon, liver, pancreatic and soft tissue cancers [24]. An increase in the ex-

Table 3. p53 expression before and after induction phase chemotherapy

\begin{tabular}{lccc}
\hline Chemotherapy & p53 expression & p-value & CI 95\% \\
\hline Before chemotherapy & $59.7 \pm 21.6$ & 0.224 & 41.6 \\
After chemotherapy & $72.3 \pm 19.9$ & & \\
\hline
\end{tabular}

Table 4. Surviving expression before and after the induction phase chemotherapy

\begin{tabular}{lccc}
\hline Chemotherapy & Surviving expression & p-value & CI 0.9\% \\
\hline Before chemotherapy & $73.4 \pm 20.6$ & 0.002 & 56.2 \\
After chemotherapy & $50.6 \pm 14.9$ & & \\
\hline
\end{tabular}

Table 5. The p53/surviving ratio before and after the induction phase chemotherapy.

\begin{tabular}{lccc}
\hline \multicolumn{1}{c}{ Chemotherapy } & p53/surviving ratio & p-value & CI 09\% \\
\hline Before chemotherapy & $0.83 \pm 0.25$ & 0.034 & 0.62 \\
After chemotherapy & $1.64 \pm 0.96$ & & \\
\hline
\end{tabular}




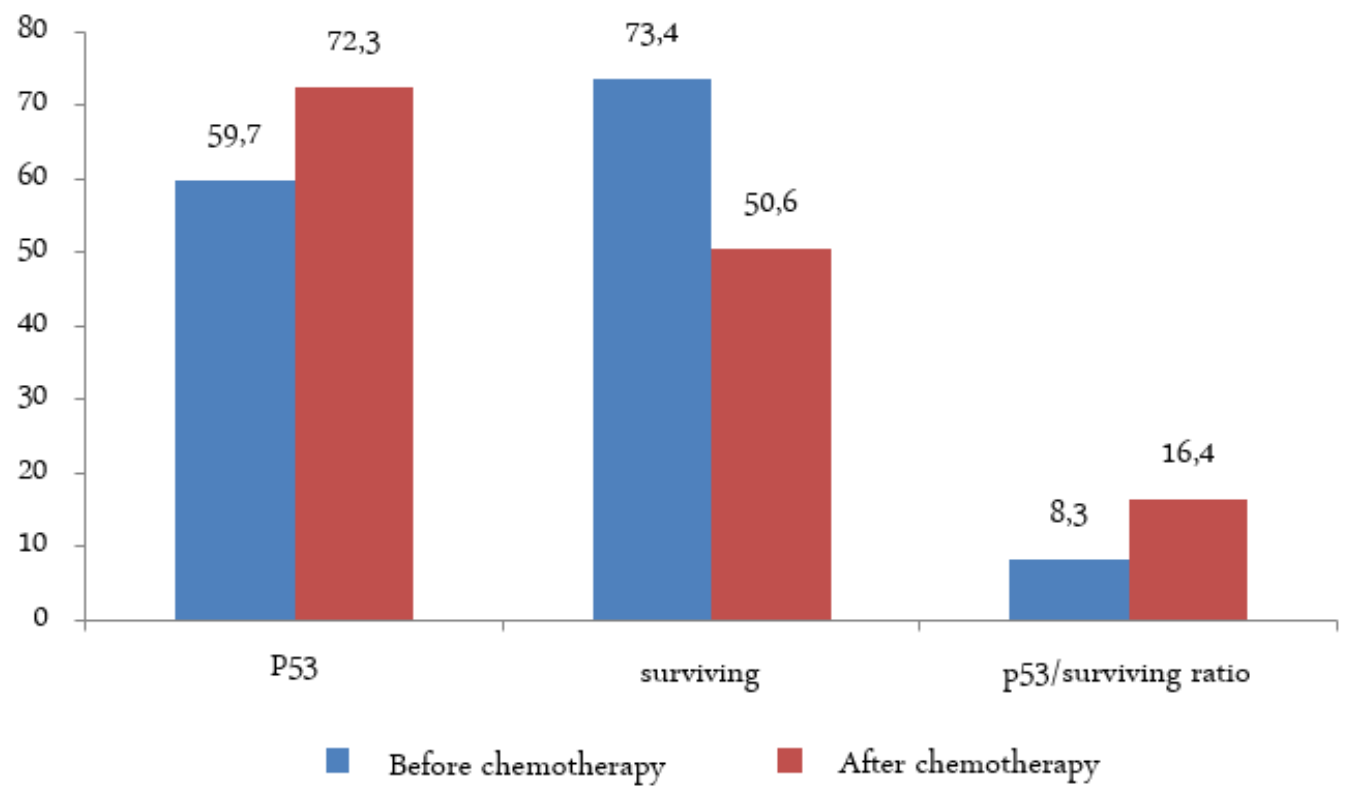

Figure 1. p53 and surviving expression and the p53/surviving ratio to induction phase chemotherapy in children with AML

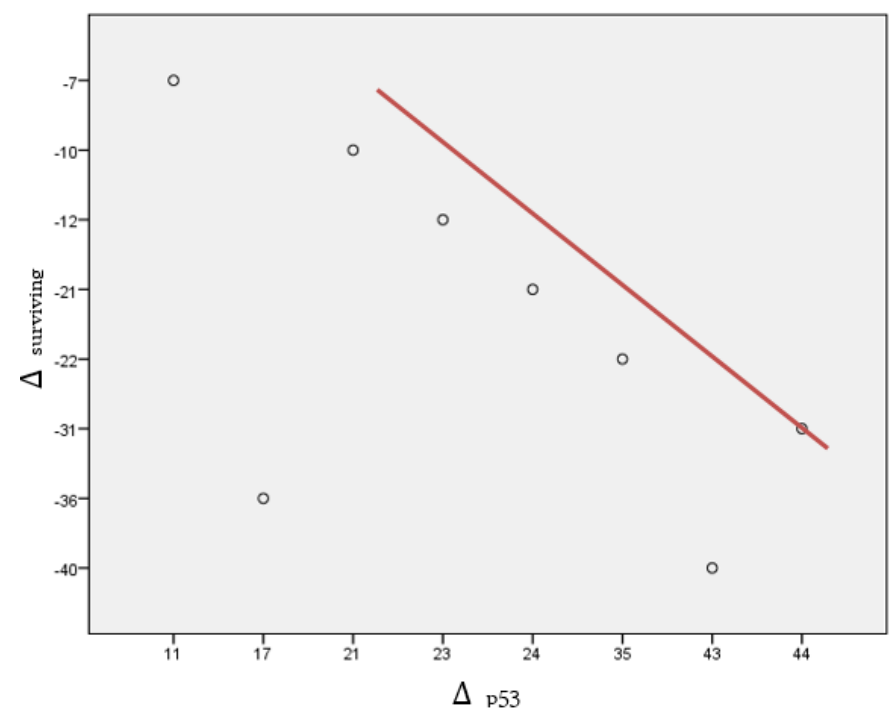

Figure 2. The Correlation between p53 and surviving expressions after the induction phase chemotherapy in children with AML

pression of surviving in various malignancies correlates with the incidence of relapse and treatment failure and the low life expectancy of patients with AML [25].

Surviving is a protein that can be expressed on a regular or cell neoplasms and controls deregulation of tumorigenicity. The regulation of surviving itself is usually dependent on cell cycles. At the time of cell proliferation, surviving can be expressed at the G2/M phase and then rapidly decreases to a resting phase (G1 phase) $[13,26]$. Furthermore, surviving is modulated to promote the cycle-dependent elements (CDE) and the cycle homology region (CHR) where the promoter is located in the transcription of G2 phase [13] $[26,27]$. Moreover, surviving can be affected by changes in RNA (siRNA) or by a suppressor surviving (YM155) where surviving will increase cell death through the p53 dependent apoptosis pathway [28]. Thus, the early detection of surviving levels will be very beneficial to both a treatment and prognosis.

Table 5 shows that the ratio of p53/surviving after the induction phase chemotherapy in AML patients increased significantly which was confirmed the hypothe- 
sis of the study.

All AML patients' response to the treatment were very varied due to prognostic factors and characteristics of AML malignancy, patients (age, clinical course and karyotype), etc. The treatment of AML has made much progress and in developed countries treatment success has reached $65 \%$ while in Indonesia is expected to remain below $10 \%[3,29]$. Apoptosis abnormalities accelerate the transformation and lead to the proliferation of tumors toward malignancy. Also, changes in the pathway of tumor suppressor genes and activation of proto-oncogenes that can play a role in cancer development especially with the deregulation of programmed cell death $[30,31]$.

Surviving levels can identify the presence of suppression by p53 (wild-type), either directly or indirectly [32] and low levels of surviving caused by the role of p53 (wild-type) dependent apoptosis. Thus, both the expression of surviving and p53 can describe a synergism regarding determining the prognosis in the case of AML in children [33, 34].

The problems in this study were when patients $\mathrm{did}$ not turn up for scheduled chemotherapy and when patients did not come for follow-up check-ups on time, then some patients dropped outs while undergoing the induction phase chemotherapy. Another problem was fatalities while undergoing chemotherapy as well as patients with absolute neutrophil counts (ANC) of less than 500 so that the necessitating improvements in their general health first. However, none of the obstacles and constraints above affected the results. The limited number of samples in this study may have affected the results statistically, but with it is hoped that they will be of use as a reference for similar studies in the future and it is also hoped that these findings will contribute to prove that the expression of p53, surviving and the P53/surviving ratio could be used as a marker in the prognosis for chemotherapy in children with AML.

\section{CONCLUSION}

Expressions of p53 did not increased significantly after the induction phase chemotherapy in children with AML, whereas the expressions of surviving decreased significantly after the induction phase. The p53/surviving ratio increased significantly after induction phase of chemotherapy in children with AML. A negative correlation was found between the p53 and surviving expressions in children with AML after induction phase chemotherapy. In this case, p53/surviving ratio can be use as parameter for induction phase chemotherapy in children with AML.

\section{ACKNOWLEDGMENT}

\section{REFERENCES}

1. Gaynon PS (2005) Childhood acute myeloid leukemia and relaps. British J Haematol 13: 579-87.

2. Spector LG, Ross JA, Robison LL (20060 Epidemiology and etiology in childhood leukemias. New York, Cambridge University Press.

3. Pui CH, Robinson LL, Look AT (2008) Acute myeloid leukemia. Lancet. 371: 1030-43.

4. Ribera JM, Oriol A (2009) Acute myeloid leukemia in adolescents and young adults. Hematol Oncol Clin North Am 23(5): 1033- 42.

5. Ross DD (2000) Novel mechanisms of drug resistance in leukemia. Leukemia 14: 467-473.

6. MacKenzie SH, Clark AC (2008) Targeting cell death in tumors by activating caspases. Current cancer drug targets 8: 98-109.

7. Altieri DC (2010) Survivin and IAP proteins in cell death mechanisms. Biochem J. 430: 199-205.

8. Schimmer AD, Pedersen IM, Kitada S, et al (2003) Functional blocks in caspase activation pathways are common in leukemia and predict patient response to induction chemotherapy. Cancer Res. 63: 1242-48.

9. Fulda S, Debatin KM (2006) Extrinsic versus intrinsic apoptosis pathways in anticancer chemotherapy. Oncogene 25: 4798-811.

10. Dohi T, Okada K, Xia F, et al (2004) An IAP-IAP complex inhibits apoptosis. J Biol Chem, 279 (33): 3408734090.

11. Carter BZ, Wang RY, Schober WD, et al (2003) Targeting survivin expression induces cell proliferasion defect and subsequent celldeath involving mitochondrial pathway in myeloid leukemic cell. Cell cycle 2 (5): 488-493.

12. Esh AM, Atfy M, Azizi NA (2011) Prognostic Significance of survivin in Pediatric Acute Lymphoblastic Leukemia.Indian J Hematol Blood Transfusion 27 (1): 18-25.

13. Prokop A, Wieder T, Sturm I et al (2000) Relaps in childhood acute lymphoblastic leukemia is associated with a decrease of the $\mathrm{Bax} / \mathrm{Bcl}-2$ ratio and loss of spontaneous caspase-3 processing in vivo. Leukemia. 14: 1606-13.

14. Mulatsih S (2009) Fusi gena TEL-AML1, BCR-ABL, E2APPBX1, dan MLL-AF4 sebagai faktor prognosis leukemia limfoblastik akut. Fakultas Kedokteran Universitas Gadjah Mada Yogyakarta.

15. Biondi, Cazzaniaga, Masera (2005) New stratification of childhood acute lymphoblastic leukemia. Hematology 1: 133-7. 
16. Holleman A, Boer ML, Kazemier MR et al (2003) Resistance to different classes of drugs is associated with impaired apoptosis in childhood acute lymphoblastic leukemia. Blood Journal Hematology 102: 4541-4546.

17. Faderl S, Thall PF, Kantarjian HM et al (1999) Caspase-2 and Caspase-3 as predictors of complete remission and survival in adults with acute myeloid leukemia. Clin Cancer Res.5: 4041-47.

18. Meyer LH, Karawajew L, Schrappe M et al (2008) Cytochrome c-related caspase- 3 activation determines treatment response and relapse in childhood precursor B-cell ALL. Blood 4524-31.

19. Ghavami S, Hashemi M, Ande SR, et al (2009) Apoptosis and cancer: mutations within caspase Genes. J Med Genet. 46: 497-510.

20. Lu Y, Chen GQ (2011) Effector caspases and leukemia. Int J Cell Biology. 10: 1-8.

21. Fulda S (2009) Therapeutic opportunities for counteracting apoptosis resistance in childhood leukemia. British J Haematol. 145: 441-54.

22. Miura M, Chen XD, et al (2004) A crucial role of caspase3 in osteogenic differentiation of bone marrow stromal stem cells. Journal of Clinical Investigation. 114(12): 17041713.

23. Darzynkiewicz Z, Huang X, Okafuji M, King M (2004) A. Cytometric methods to detect apoptosis. Methods cell biology 75: 307-341.

24. Liu T, Raetz E, Moss PJ et al (2002) Diversity of the apoptotic response to chemotheraphy in childhood leukemia. Leukemia. 16: $223-232$.

25. Alama MA, Hassab HM, Gendy WE, Zeid MY (2005) Study of caspase-3 level in relation to prognostic risk factors in children with acute myeloid leukemia. Alexandria Journal of Pediatrics. 19 (1): 45-49.
26. Soung YH, Lee JW, Kim SY (2004) Somatic mutations of CASP3 gene in human cancers. Hum Genet. 115: 112-5.

27. Shin S, Sung BJ, Cho YS et al (2001) An anti-apoptotic protein human survivin is a direct inhibitor of caspase-3 and -7. Biochemistry 40: 1117-23.

28. Tamm I, Richter S, Oltersdorf D et al (2004) High expression levels of $\mathrm{x}$-linked inhibitor of apoptosis protein and survivin correlate with poor overall survival in childhood de novo acute myeloid leukemia. Clin Cancer Res.10: 3737-44.

29. Vogler M, Giagkousiklidis S, Genze F et al (2005) Inhibition of clonogenic tumor growth: a novel function of smac contributing to its antitumor activity. Oncogene. 24: 7190202.

30. Fortugno P, Wall NR, Giodini A, et al (2002) Survivin exists in immunochemically distinct subcellular pools and is involved in spindle microtubule function. J Cell Sci 115: 575-585

31. Park E, Eun JG, Hsieh YT et al (2011) Targeting survivin overcomes drug resistance in acute lymphoblastic leukemia. Blood 118(8): 2191-2199.

32. Zhou M, Gu L, Li F et al (2002) DNA damage induces a novel p53-survivin signaling pathway regulating cell cycle and apoptosis in acute myeloid leukemia cells. J Pharmacol Exp Therapy, 303, 2002, 124-31.

33. Troeger A, Siepermann M, Escherich G et al (2007) Survivin and its prognostic significance in pediatric acute Bcell precursor myeloid leukemia. Hematologica, the hematology Journal 92 (8): 1043.

34. Yahya RS, Fouda MI, El Baz HA (2012) Serum survivin and TP 53 gene expression in children with acute myeloid leukemia. Iranian J Publ Health 41(1): 37-44. 